\title{
A new, fast and convenient method for layering blood or bone marrow over density gradient medium
}

A Islam

\begin{abstract}
A density gradient dispenser has been developed, providing a fast, easy and convenient method for layering peripheral blood or bone marrow over a density gradient solution for the isolation of lymphocytes and mononuclear cells. The present approach is just the opposite of the conventional method of layering blood over a density gradient solution. In the conventional method a required amount of density gradient solution is first placed in a centrifuge tube and then blood or marrow is layered on top using a pasteur pipette. In the present method a required amount of blood or marrow is first placed in a centrifuge tube. The required amount of density gradient solution is then gently delivered underneath the blood sample using the newly devised density gradient dispenser. Because of the difference in densities, the released density gradient solution lifts the sample of blood upwards and produces exactly the same blood and density gradient suspension as that produced by the conventional method but is less cumbersome, faster and more convenient.
\end{abstract}

(f Clin Pathol 1995;48:686-688)

Keywords: Density gradient dispenser, blood, bone marrow.

Isolation of lymphocytes and mononuclear cells from peripheral blood and bone marrow for histocompatibility testing, in vitro cell mediated immunoassays and various other procedures requires gentle and careful layering of anticoagulated blood or bone marrow on top of a synthetic high molecular weight density gradient solution such as Ficoll-Hypaque, ${ }^{1}$ Histopaque, ${ }^{2}$ Isolymph, ${ }^{3}$ or Nycomed. ${ }^{4}$ This technique is slow, tedious, often tiring, and if not precisely carried out, results in an incomplete or impure segregation of mononuclear cells.

At present there is a need for a rapid, simple, reliable, and less cumbersome approach to the isolation of mononuclear cells from blood or bone marrow aspirates using the density gra- dient solution as the separation medium. This newly developed density gradient dispenser* has been specifically designed to fulfil this requirement.

\section{Methods}

THE DISPENSER

The dispenser (fig 1 ) consists of a hollow tube made of white polycarbonate plastic (Lexan resin; General Electric, Pittsfield, Massachusetts, USA). This plastic is extensively used in various other medical devices because of its strength and integrity. The plastic is inert, and many solutions such as density gradient media and blood do not adhere to the wall of the tube due to the hydrophobic nature of the polymerised plastic resin. As a result, it does not have any untoward effects on the layering process.

The tube is $120 \mathrm{~mm}$ in length and has a uniform external diameter of $2.3 \mathrm{~mm}$ and a constant internal diameter of $2 \cdot 1 \mathrm{~mm}$. The distal portion of the tube has two notches, one on each side for the slow and controlled delivery of density gradient solution. The proximal end of the tube has been fitted with a standard female luer-lock to receive the nozzle of a syringe.

\section{PROCEDURE}

The procedure is presented in diagrammatic form in fig 2. Panel $A$-Collect $5 \mathrm{ml}$ of defibrinated or heparinised blood in a centrifuge tube (larger volumes of blood may be collected depending on the need and the density gradient manufacturer's recommended method). Panel $B$-Withdraw $4.5 \mathrm{ml}$ of the density gradient medium of choice from the container using the density gradient dispenser as shown. It is important that all air is excluded from the tip of the device as its presence within the dispenser may affect the layering process. Panel CImmerse the tip of the density gradient dispenser into the centrifuge tube containing the blood or marrow sample and gently push down

* Available from Medical Instruments and Laboratory Technologies, P.O. Box 538, Buffalo, New York 14209, USA. 


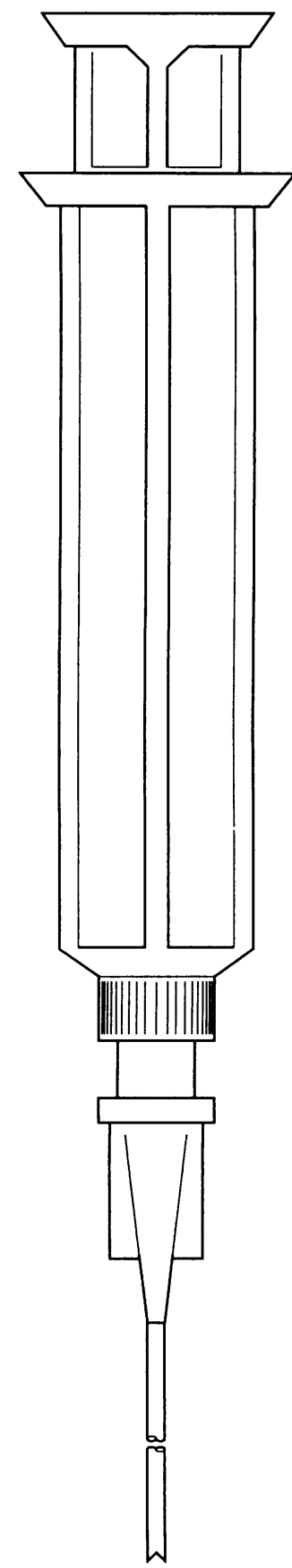

Figure 1 Diagrammatic representation of the density gradient dispenser.

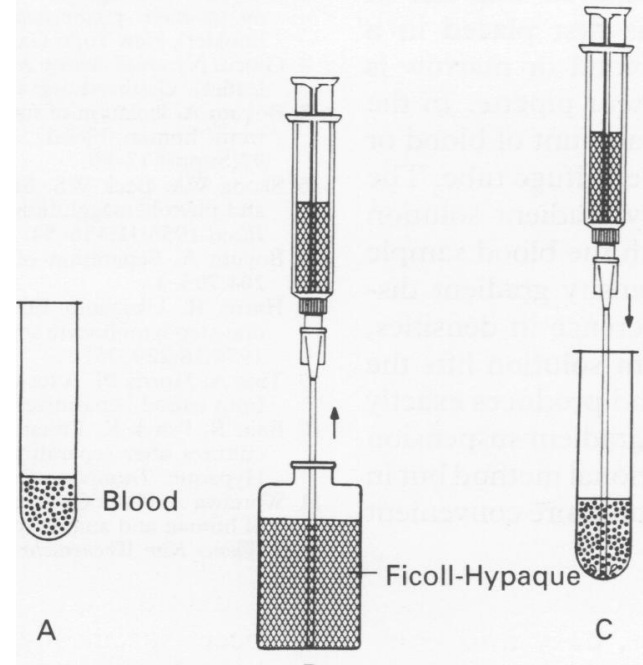

B

Figure 2 Schematic representation of the method used to separate mononuclear cells from peripheral blood or bone Figure 2 Schematic representation of the method used to separate mononuclear cells
marrow incorporating the density gradient dispenser (see text for full explanation).

the plunger of the syringe to release the density gradient solution at the bottom of the tube beneath the blood sample. As the density gradient solution is released beneath the blood sample it lifts the sample of blood upwards (panels $D$ and $E$ ). When all the density gradient solution is delivered, the sample of blood will rest on top of density gradient solution. Panel $F$ - Once the delivery of the density gradient solution is complete, the density gradient dispenser is withdrawn and the centrifuge tube containing the layered blood or marrow sample is ready for centrifugation. The remainder of the mononuclear cell isolation technique follows the established laboratory procedure or the density gradient manufacturer's recommended method.

\section{Results}

The density gradient dispenser has been extensively tested. The operators have found the design and construction of the dispenser to be satisfactory. They have also found the method fast and convenient and a considerable improvement compared with the conventional method of layering blood or bone marrow on top of Ficoll-Hypaque or some other density gradient medium using pasteur pipettes.

\section{Discussion}

Since 1964, when Boyum ${ }^{5}$ first introduced this technique, using a low viscosity density gradient solution (Ficoll-Hypaque), it has become the most widely used method for isolating lymphocytes and mononuclear cells from peripheral blood or bone marrow samples.

An early method used to separate lymphocytes from other blood cell types involved mixing the blood with an erythrocyte ag- gregating agent. This caused the erythrocytes to clump and settle at the bottom of the tube. The lymphocytes could then be collected from the upper part of the tube.$^{67}$ This technique, however, had disadvantages: lengthy and repeated procedures were required to obtain purified lymphocyte suspensions and the yield or recovery of lymphocytes was low.

Boyum first saw that the low viscosity of Ficoll-Hypaque, compared with the other polymeric erythrocyte aggregating agents, made it possible to devise a lymphocyte isolation procedure involving a short, low speed centrifugation step. ${ }^{5} \mathrm{~A}$ solution of Ficoll and sodium metrizoate of the proper density and osmotic strength was first placed in a centrifuge tube. Blood was then layered on the top of the Ficoll which remained on the top because of the density difference between the two liquids. The two phase system was centrifuged at a low speed for a short time. The erythrocytes and granulocytes sedimented to the bottom of the tube, and the purified lymphocytes could be collected from the interface between the two phases. For the first time, this procedure provided an easy one step, rapid, reproducible method for the preparation of viable lymphocytes. ${ }^{89}$

Although the above cell separation technique using a solution of Ficoll and sodium metrizoate has gained world wide acceptance, sodium diatrizoate has been successfully substituted for sodium metrizoate in recent years. ${ }^{1011}$ However, the original technique of layering defibrinated/anticoagulated blood on top of density gradient medium using a pasteur pipette has not changed. This layering technique is slow, tedious, often tiring, and if not precisely layered, yields an incomplete or impure segregation of mononuclear cells. At present, there is a need for a rapid, simple, reliable, and less cumbersome approach. 
The method described here is just the opposite of the conventional method of layering blood over a density gradient solution. In the conventional method a required amount of density gradient solution is first placed in a centrifuge tube and then blood or marrow is layered on top using a pasteur pipette. In the present method a required amount of blood or marrow is first placed in a centrifuge tube. The required amount of density gradient solution is then delivered underneath the blood sample using the newly devised density gradient dispenser. Because of the difference in densities, the released density gradient solution lifts the sample of blood upwards and produces exactly the same blood and density gradient suspension as that produced by conventional method but in a less cumbersome, faster and more convenient way.
1 Pharmacia. Ficoll-Hypaque for in vitro isolation of lymphocytes (product information leaflet). Upsala: Pharmacia LKB 1989

2 Sigma Diagnostic. Histopaque - 1077 (product information booklet). St Louis: Sigma Diagnostics, 1991.

3 Gallard-Schlesinger Industries. Isolymph: a one-step method for isolation of pure lymphocytes (production information booklet). New York: Gallard-Schlesinger Industries, 1992.

4 Gibco. Nycomed density gradient media (product information leaflet). Gaithersburg: Gibco-BRL, 1992.

5 Boyum A. Isolation of mononuclear cells and granulocytes from human blood. Scand $\mathcal{f}$ Clin Lab Invest 1968; 97(Suppl):77-89.

6 Skoog WA, Beck WS. Studies in the fibrinogen, dextran, and phytohemagglutinin methods of isolating leukocytes. Blood 1956;11:436-54

7 Boyum A. Separation of white blood cells. Nature 1964; 204:793-4.

8 Harris R, Ukaejiofo EO. Tissue typing using a routine one-step lymphocyte separation technique. Brf Haemato 1970;18:229-35.

9 Ting A, Morris PJ. A technique for lymphocyte preparation from stored heparinized blood. Vos Sang 1971;20:561-3.

10 Bain B, Pshyk K. Enhanced reactivity in mixed leukocyte cultures after separation of mononuclear cell on Ficollcultures after separation of mononuclear cell on

11 Wottawa A, Klein G, Altman H. A method for the isolation of human and animal lymphocytes with Ficoll-Urografin. Wiener Klin Wochenschr 1974;86:161-3. 